\title{
CROSSED-INVERSE AND RELATED LOOPS $\left({ }^{1}\right)$
}

\author{
BY \\ R. ARTZY
}

Introduction. Crossed-inverse (C.I.) loops are loops in which the identity $x y \cdot x^{\prime}=y$ holds for all $x, y$ of the loop, $x^{\prime}$ being the right inverse of $x$. Such loops have already been studied $[1 ; 2]$, and the results were useful in the study of neofields [6] and of geometrical subjects [3]. C.I. loops are special automorphic-inverse loops, that is, loops satisfying the identity $(x y)^{\prime}=x^{\prime} y^{\prime}$, for all $x, y$ in the loop.

The first section of this paper deals with isotopic C.I. loops, and they are proved to be also isomorphic. Then the autotopisms of C.I. loops are studied, and the main result of this part is the fact that the "companions" of all the autotopisms, i.e. the elements that correspond to the translation elements of the isotopisms, form a subloop which is commutative and Moufang. $\$ 2$ of the paper gives a necessary condition for the existence of finite automorphicinverse loops consisting only of the unit element and inverse-cycles [cf. 1] of the same length. The condition concerning the equal length of all inversecycles is necessary for the transitivity of the automorphism group of the loop [2] and hence for the possibility of considering the loop as the additive loop of a neoring. The third part deals with a method of constructing infinite automorphic-inverse loops satisfying also certain other identities.

The author is grateful to R. H. Bruck for fruitful discussions on the subject of this paper.

\subsection{Definitions.}

1.11. The biunique mapping which maps every element, $c$, of a loop $(G, \cdot)$ on its right inverse will be called $J$. Thus $c \cdot c J=1$, and $c J^{-1} \cdot c=1$. In automorphic-inverse loops $J$ is an automorphism.

1.12. In the principal isotope of the $\operatorname{loop} G$, with the new operation $(*)$ such that $a g * f b=a b$, for all $a, b$ in $G$, the ordered pair $(g, f)$ is called the pair of translation elements of the principal isotope.

\subsection{Elementary properties of C.I. loops.}

1.21. The identities $a b \cdot a J=b$ and $a(b \cdot a J)=b$ are equivalent.

Proof. If $P$ and $Q$ are biunique mappings of the loop onto itself, then $P Q=I$, the identity mapping, if and only if $Q P=I$. Let $L(x)$ and $R(x)$ be,

Presented to the Society, December 28, 1956, under the title Loops with identities, and August 30, 1957, under the title Cross-inverse loops; received by the editors August 2, 1957.

(1) Supported in part by the Research Committee of the Graduate School of the University of Wisconsin from funds supplied by the W. A. R. F. 
respectively, the usual denotations for left and right multiplication by $x$. Then $L(a)$ and $R(a J)$ are biunique mappings of the loop onto itself, and $L(a) R(a J)=I$ is equivalent to $R(a J) L(a)=I$.

1.22. From the C.I. property follows the automorphic-inverse property, but the converse statement is not true.

Proof. For the first part see [1]. The second part is proven by the existence of easily constructed counter examples.

1.23. Isotopes of C.I. loops are not necessarily C.I.

EXAmple. The following C.I. loop, with translation elements 2 and 3, yields a principal isotope which is not C.I.

$\begin{array}{lllll}1 & 2 & 3 & 4 & 5 \\ 2 & 1 & 4 & 5 & 3 \\ 3 & 5 & 1 & 2 & 4 \\ 4 & 3 & 5 & 1 & 2 \\ 5 & 4 & 2 & 3 & 1\end{array}$

If we denote the $J$-automorphism of 1.11 in the principal isotope by $J^{*}$, we get, e.g., $(3 * 4) * 3 J^{*}=3$, instead of 4 .

1.24. If in a C.I. loop $G$ an element belongs to one of the (right, left or middle) nuclei, $A_{\rho}, A_{\lambda}, A_{\mu}$, respectively, then it belong to the centre, $Z$.

Proof. Let $z \in A_{\rho}$, that is

$$
a \cdot b z=a b \cdot z
$$

all $a, b \in G$.

Equation (1) implies, with $a=d J^{-1}$ and $b=c d$,

$$
\begin{aligned}
d J^{-1}(c d \cdot z) & =\left(d J^{-1} \cdot c d\right) z, \\
d J^{-1}(c d \cdot z) & =c z, \\
c d \cdot z & =c z \cdot d .
\end{aligned}
$$

Putting $c=1$ yields $d z=z d$, for all $d$ in $G$. Using this, we get $z \cdot c d=z c \cdot d$, and hence $z \in A_{\lambda}$. Now apply (1) in (2), and, in view of $x z=z x$ for all $x$ in $G$, we get $c \cdot d z=c \cdot z d=c z \cdot d$, and hence $z \in A_{\mu}$.

If $z \in A_{\lambda}$ is assumed, the proof of $x z=z x$, all $x$ in $G$, and $z \in A_{\rho}$ and $z \in A_{\mu}$ is analogous.

Now let $z \in A_{\mu}$, that is $a z \cdot b=a \cdot z b$ for all $a$ and $b$ in $G$. Put $a=z J^{-1} \cdot c J^{-1}$ and $b=d c$. We get

$$
\begin{aligned}
\left(\left(z J^{-1} \cdot c J^{-1}\right) z\right) \cdot d c & =\left(z J^{-1} \cdot c J^{-1}\right)(z \cdot d c), \\
d & =\left(z J^{-1} \cdot c J^{-1}\right)(z \cdot d c) .
\end{aligned}
$$

$d \cdot z c=z \cdot d c$, and since for $c=1$ we get $x z=z x$ for all $x$ in $G$, this becomes $d \cdot c z=d c \cdot z$, and hence $z \in A_{\rho}$. In view of the first part of the proof, this means $z \in Z$. 


\subsection{Isotopic C. I. loops.}

Lemma 1. If a principal isotope of a C.I. loop $G$ is again a C.I. loop, and if the principal isotope has the translation elements $g$ and $f$, then

$$
f a \cdot g b=f g \cdot a b=(f \cdot a b) g=f(g \cdot a b), \quad \text { all } a, b \text { in } G .
$$

Proof. The C.I. property implies $R(x)^{-1}=L\left(x J^{-1}\right)$ and $L(x)^{-1}=R(x J)$ for all $x$ in $G$. The unit element of the principal isotope is $f g$. For the right inverse of $c$ in the principal isotope, $c J^{*}$, we have $c * c J^{*}=f g$,

$$
\begin{aligned}
\left(g J^{-1} \cdot c\right)\left(c J^{*} \cdot f J\right) & =f g, \\
c J^{*}=f\left((f g)\left(g J^{-1} \cdot c\right) J\right) & =f((f g)(g \cdot c J)) .
\end{aligned}
$$

Thus $J^{*}=J L(g) L(f g) L(f)$. Now, if the principal isotope is C.I., we have $c *\left(d * c J^{*}\right)=d$,

$$
c L\left(g J^{-1}\right) \cdot\left(d L\left(g J^{-1}\right) \cdot c J L(g) L(f g) L(f) R(f J)\right) R(f J)=d .
$$

Now the C.I. property implies $L(f) R(f J)=I$, the identity mapping. Thus

$$
\begin{aligned}
c L\left(g J^{-1}\right) \cdot\left(d L\left(g J^{-1}\right) \cdot c J L(g) L(f g)\right) R(f J) & =d, \\
d L\left(g J^{-1}\right) \cdot(c J L(g) L(f g)) & =\left(d \cdot c L\left(g J^{-1}\right) J\right) R(f J)^{-1}, \\
& =(d \cdot c J L(g)) L(f) .
\end{aligned}
$$

Put $c J L(g)=a b$ and $d=b J^{-1}$. Then we get

$$
\begin{aligned}
(g b) J^{-1}(f g \cdot a b) & =f\left(b J^{-1} \cdot a b\right)=f a, \\
f g \cdot a b & =f a \cdot g b .
\end{aligned}
$$

Equation (4) with $a=1$ and $b=e$ yields

$$
f g \cdot e=f \cdot g e,
$$

and by setting $b=1$ and $a=e$ in (4) we have

$$
f g \cdot e=f e \cdot g \text {. }
$$

Apply now identities (5) and (6), with $a b$ in place of $e$, in (4).

$$
f a \cdot g b=f(g \cdot a b)=(f \cdot a b) g .
$$

LEMma 2. Under the assumptions of Lemma 1, the following relations hold:

(i) $f g=g f$, (ii) $g J^{2}=g$, (iii) $f J^{2}=f$.

Proof. Since the isotope is C.I., we have identically $(c * d) * c J^{*}=d$, that is,

$$
\left(c L\left(g J^{-1}\right) \cdot d R(f J)\right) L\left(g J^{-1}\right) \cdot c J L(g) L(f g) L(f) L(f)^{-1}=d .
$$

Put $c J L(g)=a$ and $b J^{-1}=d$; then this becomes $\left(a J^{-1} \cdot b J^{-1} R(f J)\right) L\left(g J^{-1}\right)$ $\cdot a L(f g)=b J^{-1}$, or, because of the automorphic-inverse property, 


$$
\left(a \cdot b R\left(f J^{2}\right)\right) L(g) \cdot a J L((f g) J)=b,
$$

or

$$
g\left(a\left(b \cdot f J^{2}\right)\right)=(f g \cdot a) b .
$$

With $a=b=1$ this becomes

$$
g \cdot f J^{2}=f g .
$$

Equation (3) for the case $g \cdot c J=g J$ and $g \cdot d J=1$ becomes $f g \cdot g J=f$, or

$$
f g=g f \text {. }
$$

Comparison of (9) and (10) yields $f J^{2}=f$, and as a consequence, $f J=f J^{-1}$ $=f^{-1}$. Equation (8) becomes now $g(a \cdot b f)=(f g \cdot a) b$. With $b=g$ and $a=g J^{-1}$ this becomes $g\left(g J^{-1} \cdot f g\right)=\left(f g \cdot g J^{-1}\right) g$, and in view of $(10), g f=\left(f g \cdot g J^{-1}\right) g$ or $f=g f \cdot g J^{-1}$. The C.I. property yields $f=g f \cdot g J$; thus $g J=g J^{-1}=g^{-1}$, and hence $g=g J^{2}$.

Theorem 1. Isotopic C.I. loops are isomorphic.

Proof. We have from Lemma 1 the relation $f a \cdot g b=(a b) L(f) R(g)$. Now $f a=a L(f) R(g) R(g)^{-1}$, and $g b=(f \cdot b L(g)) f^{-1}$, which, in view of the combined equalities (5) and (6), equals $(f b \cdot g) f^{-1}=b L(f) R(g) L(f)^{-1}$. Now call $L(f) R(g)$ $=T$. Then is $a T * b T=a T R(g)^{-1} \cdot b T L(f)^{-1}=(a b) T$, and $T$ is an isomorphism as required. (The present form of this proof has been influenced by a private communication by Shmuel Schreiber.)

Theorem 2. A principal isotope of a C.I. loop is C.I. if and only if the identity $f a \cdot g b=f g \cdot a b$ holds in the loop, $g$ and $f$ being the translation elements of the principal isotope.

Proof. The necessity follows from Lemma 1.

Put in (4) $b=d J$ and $a=d \cdot c J L(g)$, so that $a b=c J L(g)$. Then $(d \cdot c J L(g)) L(f) \cdot d J L(g)=c J L(g) L(f g), \quad d \cdot c J L(g)=\left(d L\left(g J^{-1}\right) \cdot c J L(g) L(f g)\right)$ $R(f J), d=c L\left(g J^{-1}\right) \cdot\left(d L\left(g J^{-1}\right) \cdot c J L(g) L(f g)\right) R(f J)=c *\left(d * c J^{*}\right)$, which implies the C.I. property for the isotope.

1.4. Some useful identities. In the following, $g$ and $f$ will always denote the translation elements of a principal isotope, and $a, b, c, d$ will be arbitrary elements of a C.I. loop.

1.41. Put, with modifications implied by Lemma 2, in (3) $a=g^{-1} d$ and $b=g \cdot c J$. We get then

$$
a(f g \cdot b)=f(a g \cdot b)
$$

and with $b=1$,

$$
a \cdot f g=f \cdot a g
$$


1.42. Equality (4) yields the autotopism $(L(f), L(g), L(f g))$. Now $(J, J, J)$ and $\left(J^{-1}, J^{-1}, J^{-1}\right)$ are automorphisms and therefore also autotopisms. Hence

$$
(J, J, J)(L(f), L(g), L(f g))^{-1}\left(J^{-1}, J^{-1}, J^{-1}\right)=(R(f), R(g), R(f g))
$$

is another autotopism, and

$$
a f \cdot b g=a b \cdot f g \text {. }
$$

With $b=1$ this becomes

$$
a f \cdot g=a \cdot f g .
$$

Apply (14) to (13) and get

$$
a f \cdot b g=(a b \cdot f) g .
$$

1.43. In (11) substitute $c=a J \cdot g J$ and $d=(g \cdot b f) J$, that is, $a=g J \cdot c J^{-1}$ and $b=(f \cdot d g) J^{-1}$. The result is $\left(g J \cdot c J^{-1}\right)\left(f g \cdot(f \cdot d g) J^{-1}\right)=f\left(c J^{-1} L(g J) R(g)\right.$ $\left.\cdot(f \cdot d g) J^{-1}\right)=f(c(f \cdot d g)) J^{-1}$, and $g c \cdot\left(f^{-1} g^{-1} \cdot(f \cdot d g)\right)=f J \cdot(c(f \cdot d g))$. We have from (4) $g c \cdot d=f J \cdot(c(f \cdot d g))$ and $(g c \cdot d) f=c(f \cdot d g)$. Set here $c=1$ and then $d=1$, and apply the resulting identities to the equality itself. We get $(g c \cdot d) f$ $=c(d \cdot f g)$, and with $d=1$,

$$
g c \cdot f=c \cdot f g .
$$

1.5. Autotopisms of C.I. loops. Equation (4) implies that

$$
\tau=(L(f), L(g), L(f g))
$$

is an autotopism if $a g * f b=a b$ determines a principal isotope with the C.I. property. Let $(U, V, W)$ be any autotopism. Then $1 U \cdot a V=a W$, and $a U \cdot 1 V$ $=a W$, for every loop element $a$, hence

$$
V=W L(1 U)^{-1}=W R(1 U J), \quad U=W R(1 V)^{-1}=W L\left(1 V J^{-1}\right) .
$$

The ordered pair $(1 \mathrm{~V}, 1 \mathrm{U})$ will be called the companion couple of the autotopism $(U, V, W), 1 \mathrm{~V}$ the left companion, $1 \mathrm{U}$ the right companion, $1 \mathrm{~V} \cdot 1 \mathrm{U}$ the companion product. The autotopism $\tau$ has the companion couple $(g, f)$. Thus the companion couple coincides with the translation elements of the principal isotope which led to the autotopism $\tau$. The converse is also true:

TheOREM 3. Every autotopism of a C.I. loop $G$ determines uniqucly a C.I. principal isotope with translation elements equal to the companions of the autotopism.

Proof. If the autotopism is $(U, V, W)$, let $1 V=g, 1 U=f$. Then (17) becomes $V=W R(f J), U=W L\left(g J^{-1}\right)$, and hence $a W L\left(g J^{-1}\right) \cdot b W R(f J)=(a b) W$. From the C.I. property follows now $a W R(g)^{-1} \cdot b W L(f)^{-1}=(a b) W$, and we obtain the principal isotope with $a W * b W=(a b) W$ and $x g * f y=x y$, for all $a, b, x, y$ in $G$. 
THEOREM 4. In a C.I. loop $G$ the left companions, the right companions and the companion products of the autotopisms form respective subloops of $G$. These loops are identical to each other.

Proof. Let $(U, V, W)$ be an autotopism. We have $a U \cdot b V=(a b) W$, for all $a, b$ in $G$, and hence $b V=(a b) W \cdot a U J=(a b) W \cdot(a J) J^{-1} U J$. Thus

$$
\left(W, J^{-1} U J, V\right)
$$

is another autotopism, and by repetition of the process, also

$$
\left(V, J^{-1} W J, J^{-1} U J\right) \text {. }
$$

Another autotopism is

$$
\left(V, J^{-1} W J, J^{-1} U J\right)^{-1}\left(W, J^{-1} U J, V\right)=\left(V^{-1} W, J^{-1} W^{-1} U J, J^{-1} U^{-1} J V\right)=\theta,
$$

say. By substituting $a=1$ and $b=1$ in $a U \cdot b V=(a b) W$, we get, respectively, $V^{-1} W=L(1 U)$ and $U^{-1} W=R(1 V)$. Hence

$$
\theta=\left(L(1 U), J^{-1} R(1 V)^{-1} J, J^{-1} U J V\right)=\left(L(1 U), L(1 V), J^{-1} U J V\right) .
$$

Thus $(1 U \cdot a)(1 V \cdot b)=(a b) J^{-1} U J V=(a b) S$, say. For the case $a=1$ we find $S=L(1 V) L(1 U)$. Hence $(1 U \cdot a)(1 V \cdot b)=1 U(1 V \cdot a b)$. Now put $a=1 V$ and $b=c R(1 V J)$, then we get $(1 U \cdot 1 V)(1 V \cdot c R(1 V J))=1 U(1 V \cdot c R(1 V J))$ and $(1 U \cdot 1 V) c=1 U(1 V \cdot c)$. Applying this relation with $c=a b$, we get $(1 U \cdot a)$ $\cdot(1 V \cdot b)=(1 U \cdot 1 V) \cdot a b$.

Now let $\left(U_{i}, V_{i}, W_{i}\right), i=1,2$, be autotopisms, $1 V_{i}=g_{i}, 1 U_{i}=f_{i}$. Then $\left(L\left(f_{i}\right), L\left(g_{i}\right), L\left(f_{i} g_{i}\right)\right)$ are autotopisms. Their product is

$$
\left(L\left(f_{1}\right) L\left(f_{2}\right), L\left(g_{1}\right) L\left(g_{2}\right), L\left(f_{1} g_{1}\right) L\left(f_{2} g_{2}\right)\right),
$$

and since $1 L\left(f_{1}\right) L\left(f_{2}\right)=f_{2} f_{1}$ and $1 L\left(g_{1}\right) L\left(g_{2}\right)=g_{2} g_{1}$, also $\left(f_{2} f_{1} \cdot a\right)\left(g_{2} g_{1} \cdot b\right)$ $=\left(f_{2} f_{1} \cdot g_{2} g_{1}\right) \cdot a b$. Thus $\left(f_{2} f_{1}, g_{2} g_{1}\right)$ is a companion couple. If $(g, f)$ is a companion couple, then by the automorphic-inverse identity, also $(g J, f J)$ is a companion couple. As a consequence of (18) and (19), the existence of the autotopism $(L(f), L(g), L(f g))$ implies the existence of the autotopisms $(L(f g), L(f J), L(g))$ and $(L(g), L(f J \cdot g J), L(f J))$. Therefore, if any element appears as left or right companion or as companion product, it appears also in the other two roles $\left({ }^{2}\right)$.

Definition. The subloop consisting of all the companions of $G$ is called the companion nucleus of $G$.

All the centre elements of $G$ belong, of course, to the companion nucleus $C$, and the centre $Z$ is a normal subloop of $C$. In the following, a fact about $C / Z$ will be proved.

(2) The statement in Bull. Amer. Math. Soc. Abstract 63-6-621 that the companions form a normal subloop was erroneous. 
THEOREM 5. Every element of $C$, appearing as right or left companion or as companion product, determines the two other elements uniquely, up to multiplication by centre elements. If at least one element of the triple is a centre element, so are the rest.

Proof. (i) Let $(g, f),(w, f z)$ be two companion couples with equal companion products. Then $w=f g \cdot(f z) J=f g \cdot(f J \cdot z J)$. By (4) we have $w=g \cdot z J$. Another couple is then $(w \cdot g J, f z \cdot f J)=(z J, z)$. Since $z$ is a companion, $z J=z^{-1}$. Now $z a \cdot z^{-1} b=a b$, and with $a=1, z \cdot z^{-1} b=b$. Because of $z^{-1} b \cdot z=b$, we have $z^{-1} b=b z^{-1}$, and therefore $z x=x z$ for all $x$ in $G$. By (8), with the companion couple $\left(z^{-1}, z\right)$, we get $z^{-1}(c \cdot d z)=c d$, and $c \cdot d z=c d \cdot z$. Hence $z$ belongs to the centre.

(ii) Let $(g, f)$ and $(g, f z)$ be two companion couples. Then $\left(g g^{-1}, f z \cdot f J\right)$ $=(1, z)$ is a companion couple, and $z a \cdot b=z \cdot a b$. Thus $z$ is a centre element. The same treatment applies, of course, to companion couples $(g, f)$ and $(g z, f)$.

(iii) Let $z$ be in $Z,(z, f)$ a companion couple. The equality $f a \cdot z b=f z \cdot a b$ implies $f a \cdot b=f \cdot a b$, and $f$ and $f z$ belong to $Z$. Let now $(g, f)$ be a companion couple with $f g$ in $Z$. Then $(1, f g)$ is another companion couple, and (i) implies $f g \cdot f J=g \in Z$; also $(f g, 1)$ is a companion couple, and $g^{-1} \cdot f g=f \in Z$. This completes the proof.

A well known fact about commutative Moufang loops [cf. 4] follows readily from Theorem 5 .

Corollary. In commutative Moufang loops the cubes of all elements are in the centre.

Proof. A commutative Moufang loop is C.I., and its companion nucleus is the whole loop since each element $x$ satisfies the identity $x a \cdot x b=x^{2} \cdot a b$. In view of (18) and (19), from the autotopism $\left(L(x), L(x), L\left(x^{2}\right)\right)$ other autotopisms $\left(L\left(x^{2}\right), L(x J), L(x)\right)$ and $\left(L(x J), L\left(x^{2}\right), L(x)\right)$ can be derived. Then, applying the method of the proof of Theorem 5, (i), on these two autotopisms and using the power associativity of Moufang loops, we get $x^{2} \cdot\left(x^{-1}\right)^{-1}=x^{3} \in Z$.

R. H. Bruck mentions [4] three special types of autotopisms of I.P. loops. The corresponding types are here (i) $(T, T, T)$ where $T$ is an automorphism, (ii) $(L(f), L(g), L(f g))$ where $(g, f)$ is a companion couple, (iii) $(R(z), I, R(z)$ ) where $I$ is the identity mapping and $z$ is a centre element. From each of these types additional autotopisms may be derived by means of (18) and (19). As in the case of commutative I.P. loops, which are special C.I. loops, we can prove the following theorem, thus generalizing the corresponding theorem $[4$, p. 300$]$.

THEOREM 6. The autotopism group of a C.I. loop is generated by the autotopisms of the three types mentioned above: every autotopism has the form $\alpha \beta \gamma$ where $\alpha, \beta, \gamma$ are autotopisms of the types (i), (ii), (iii), respectively. 
Proof. Let $(U, V, W)$ be an autotopism, $1 U=f, 1 V=g$. Then, by (17), $(U, V, W)=(W L(g J), W R(f J), W)$. Now put $W R(f J \cdot g J)=T$. Then, by (16), $W L(g J)=W R(f J \cdot g J) L(f)$, and, by (14), $W R(f J)=W R(f J \cdot g J) L(g)$. Thus

$$
(U, V, W)=(T L(f), T L(g), T L(f g)) .
$$

Let now $z$ be any centre element; then $L(f \cdot z J) R(z)=L(f)$, and $L\left(f z^{-1} \cdot g\right) R(z)$ $=L(f g)$. Hence

$$
(U, V, W)=\left(T L(f \cdot z J) R(z), T L(g), T L\left(f z^{-1} \cdot g\right) R(z)\right) .
$$

$(U, V, W)$ and $(L(f), L(g), L(f g))$ are autotopisms, and so is $(T, T, T)$ in view of (20), and $T$ is an automorphism. Now $\left(L(f \cdot z J), L(g), L\left(f z^{-1} \cdot g\right)\right)$ is an autotopism because $\left(f z^{-1} \cdot a\right)(g b)=\left(f z^{-1} \cdot g\right) \cdot a b$ follows from (4) and from the fact that $z$ lies in $Z ;\left(f z^{-1}, g\right)$ is the companion couple of this autotopism. Now (21) can be written in the form

$$
(U, V, W)=(T, T, T)\left(L(f \cdot z J), L(g), L\left(f z^{-1} \cdot g\right)\right)(R(z), I, R(z))=\alpha \beta \gamma .
$$

REMARK. In $\$ 1.6$ we shall prove that for the type (ii) of Theorem 6 always $f=g$. Thus the autotopism $\beta$ is necessarily of the form $\left(L(x), L(x), L\left(x^{2}\right)\right)$.

1.6. Companion loops. A loop fulfilling the requirements of the companion nucleus of a C.I. loop will be called a companion loop, $A$. We define it as a C.I. loop with the property that for every $g$ in $A$ there is an $f$ in $A$ determined uniquely to within multiplication by centre elements, such that $f y \cdot g z=f g \cdot y z$ for all $y$ and $z$ in $A$. If we define a mapping $\phi$ by $f=g \phi$, the companion couples are of the form $(x, x \phi)$, and in view of Theorem $4, \phi$ is an automorphism of $A / Z$. Moreover $x \phi^{2}=(x \phi \cdot x)^{-1}$, and $x \phi^{3}=x$, for all $x$ in $A / Z$; hence $\phi$ is of order 3 or 1 .

LEMma 3. Every inner mapping of a companion loop is an automorphism.

Proof. Since the mapping $\phi$ is defined only up to multiplication by centre elements it should be understood in the proofs of this lemma and of Theorem 7 , that for each of the expressions $c \phi$ or $c \phi^{-1}$ any of the possible values may be chosen. But once chosen, it must be kept fixed. Furthermore the choice must be restricted so that $1 \phi=1$ and $\phi \phi^{-1}=I$.

Let now, for certain $a_{1}, a_{2}, \cdots, a_{n}$ in $A, S=\prod_{i=1}^{n} V_{i}\left(a_{i}\right)$, where $V_{i}$ is either $L$ or $R$. Let $T=\prod_{i=1}^{n} W_{i}$, where $W_{i}=R\left(a_{i} \phi\right) R\left(a_{i}\right)$ in case $V_{i}=R$, and $W_{i}=L\left(a_{i}\right) L\left(a_{i} \phi\right)$ in case $V_{i}=L$. Then repeated application of (7) and (15) yields $x \phi^{-1} S \phi=(x y) T$, for all $x$ and $y$ in $A$. Now assume $S$ to be an inner mapping, i.e. $1 S=1$. Then $x=1$ yields $y S=y T$. Put now $y=1$, and get $x \phi^{-1} S \phi=x T$. Thus $S=\phi^{-1} S \phi=T$, and therefore $x S \cdot y S=(x y) S$. Thus $S$ is an automorphism of $A$. 
In the following lemma an associativity property of companion loops will be given.

Lemma 4. If $a, b, c$ are fixed arbitrary elements of $A / Z$ then $a b \cdot c=a \cdot b c$ implies $c \phi J \cdot a b=(c \phi J \cdot a) b$.

Proof. Write $(c \phi J \cdot a b) \cdot c \phi$ instead of $a b$, and $(c \phi J \cdot a) \cdot c \phi$ instead of $a$. Then is $((c \phi J \cdot a b) \cdot c \phi) c=((c \phi J \cdot a) \cdot c \phi) \cdot b c$, which becomes, by (13) and (15),

$$
(c \phi J \cdot a b)(c \phi \cdot c)=((c \phi J \cdot a) b)(c \phi \cdot c) \text { and } c \phi J \cdot a b=(c \phi J \cdot a) b .
$$

We are now able to prove the main theorem. In the proof some steps are borrowed from [5].

Theorem 7. Every companion loop $A$ is commutative and Moufang.

Proof. Let, for fixed elements $a$ and $b, S=L(a b) R(a J) R(b J)$. Then $S$ is an inner mapping and, by Lemma 3 , an automorphism. It leaves fixed all elements $z$ for which $a b \cdot z=a \cdot b z$. The $z$ 's form a subloop $H$ of $A$ because $x S=x$, $y S=y$ imply $(x y) S=x y$; and $x S=x, y=x J$ imply $x \cdot x J S=1 S$ and hence $x J=x J S$. Now $a$ is in $H$ because $a b \cdot a J=a(b \cdot a J)$. Furthermore $b \phi^{-1}$ is in $H$ because $a b \cdot b \phi^{-1}=a\left(b \cdot b \phi^{-1}\right)$ by (14). Thus also $a \cdot b \phi^{-1}$ is in $H$, which means $a b \cdot\left(a \cdot b \phi^{-1}\right)=a\left(b\left(a \cdot b \phi^{-1}\right)\right)$. Apply (13) to the left hand side and (12) to the right hand side, and get $a^{2}\left(b \cdot b \phi^{-1}\right)=a\left(a\left(b \cdot b \phi^{-1}\right)\right)$. But $b \cdot b \phi^{-1}=b \phi J$, and thus $a^{2} \cdot b \phi J=a(a \cdot b \phi J)$. Application of Lemma 4 to this equality yields $b \phi^{2} \cdot a^{2}$ $=\left(b \phi^{2} \cdot a\right) a$. Now let $K$ be the subloop consisting of all $w$ with $\left(b \phi^{2} \cdot a\right) w$ $=b \phi^{2} \cdot a w$. Then $a$ lies in $K$, and so does $a J$. Thus we have $\left(b \phi^{2} \cdot a\right) \cdot a J=b \phi^{2}$. In view of the C.I. property $a$ and $b \phi^{2}$ commute. But, since $a$ and $b$ were chosen arbitrarily the loop is commutative and therefore an I.P. loop. Now, using Lemma $4 \mathrm{C}$ of $[4$, p. 296], we see that the autotopism

$$
(L(y), L(x), L(y x))
$$

implies the identity $y a \cdot b y=(y \cdot a b) y$, and $y$ is a Moufang element. But, since in a companion loop every element can appear as right companion $y$, all its elements are Moufang elements, and the loop is Moufang.

Corollary 1. One companion of an autotopism of a C.I. loop is equal to the product of the other companion and a centre clement.

Corollary 2. In type (ii) of Theorem $6, f=g$.

2. Restrictions on the order of certain finite loops. In this part we are going to establish a necessary condition for finite automorphic-inverse loops to have a transitive automorphism group; their inverse-cycles are consequently [cf. 2] of the same length. As in [1], an inverse-cycle is defined as consisting of all $a J^{k}, k=0,1, \cdots, n-1$, if $a J^{n}=a$. 
THEOREM 8. If an automorphic-inverse loop $G$ consists only of the unit element and $r$ inverse-cycles of equal length, then $n$ divides $r^{2} n(n-1) / 2-r$.

Proof. Let $a_{1}, \cdots, a_{r}$ be $r$ nonunit elements of $G$, no two of them from the same inverse-cycle. Then $a_{m} J^{h}$, with $m=1, \cdots, r$ and $h=0, \cdots, n-1$, ranges through all nonunit elements of $G$. Now let $k$ be any integer, $0<k \leqq r$; then for any fixed $k, m, h$, there are uniquely determined $q$ and $p$ such that

$$
a_{q} J^{p} \cdot a_{k}=a_{m} J^{h},
$$

except in the cases $k=m$ where $h$ has to be assumed to be nonzero. Let now $k, m, h$ run independently through all possible values. Then, with $h$ ranging through $n$ values and $k$ and $m$ each ranging through $r$ values, but with $r$ equations with $h=0$ excluded, there are $r^{2} n-r$ distinct equations (22). We sum now the $h$ 's of all these equations:

$$
\sum h=r^{2}(0+1+\cdots+n-1)-r \cdot 0=r^{2} n(n-1) / 2 .
$$

Using the automorphic-inverse property we can write (22) in another form:

$$
a_{q} \cdot a_{k} J^{-p}=a_{m} J^{h-p} .
$$

Since each Equation (22) yielded a unique Equation (24) and since also the converse is true, we have here again $r^{2} n-r$ distinct equations. These are all possible equations of the type $a_{i} b=c$, where $b$ and $c$ are nonunit loop elements, and $a_{i}$ is defined as above. Hence $q$ ranges through $1,2, \cdots, r$, and for each of its values, $a_{k} J^{-p}$ runs through all the $r n$ nonunit loop elements except $a_{q} J^{1}$, because in that case the right hand side would become 1 and could not be expressed as $a_{m} J^{h-p}$. Now we sum $(\bmod n)$ the $-p$ over all the $r^{2} n-r$ equations (24) and get

$$
\begin{aligned}
\sum(-p) & \equiv r(r(0+1+\cdots+n-1)-1) \\
& \equiv r^{2}(n-1) / 2-r(\bmod n) .
\end{aligned}
$$

The right hand side of (24) ranges in the $r^{2} n-r$ distinct equations through the nonunit elements, once for each value of $q$ in the first term of the left hand side, $a_{q}$. The only loop element which $a_{m} J^{h-p}$ cannot equal for a given $q$ is $a_{q} J^{0}=a_{q}$ because $a_{k} J^{-p} \neq 1$. Now we sum $(\bmod n)$ the $h-p$ for all the $r^{2} n-r$ equations (24) and get

$$
\left.\sum(h-p) \equiv r(r(0+1+\cdots+n-1)-r \cdot 0)\right) \quad(\bmod n),
$$

or $\sum h+\sum(-p) \equiv r^{2} n(n-1) / 2(\bmod n)$. Combining this congruence with (23) and (25) we have $r^{2} n(n-1) / 2+r^{2} n(n-1) / 2-r \equiv r^{2} n(n-1) / 2(\bmod$ $n), r^{2} n(n-1) / 2-r \equiv 0(\bmod n)$. This completes the proof.

For $n=1$ and $n=2$ the requirement of Theorem 8 is always fulfilled. For the more interesting case $n>2$ (because then I.P. loops and groups are excluded) the smallest possible loop orders are 10 , with $n=r=3$, and 17 , with 
$n=r=4$. For these cases the author has constructed multiplication tables; in the first case the constructed loop cannot be C.I., as can be proved. In the second case actually constructed examples show that the loop may be C.I. but is not necessarily C.I.

The condition of Theorem 8 implies the condition $n \mid 2 r$, which was mentioned in [1], but the condition of the present theorem is stronger, as can be easily verified for the example $n=4, r=6$.

Added in proof. In a more recent paper by Toshiharu Ikuta (Nat. Sci. Rep. Lib. Arts Sci. Fac. Shizuoka Univ. vol. 2 (1957) pp. 1-4) appears a theorem equivalent to Theorem 8: Under the same assumptions, $r \equiv 0(\bmod n)$ for even $r, 2 r \equiv 0(\bmod n)$ for odd $r$.

3. Construction of infinite automorphic-inverse loops. In the following, a method of construction will be described; each of the constructed automorphic-inverse loops satisfies also another identity. The method is an elaboration and generalization of the technique used for the construction of C.I. loops in [1].

Let the infinite multiplicative group $\{J\}$ serve as the multiplicative group of a neofield $(N,+, \cdot)$. Our aim is to construct stepwise the loop $(N,+)$. In addition to the powers of $J$, including $J^{0}=1, N$ contains only the element 0 with the rules $x \cdot 0=0 \cdot x=0$ and $x+0=0+x=x$, all $x$ in $N$. We postulate $J^{n}+J^{n+1}=0$ for all integers $n$. Thus $J$ has now in $(N,+)$ the same meaning as in 1.11, and the distributive law of $(N,+, \cdot)$ signifies the automorphicinverse property of $(N,+)$.

Let the function $\theta$ be defined by $J^{0}+J^{k+1}=J^{k \theta}$ for all nonzero integers $k$. Thus $J^{a}+J^{b}=J^{a+(b-a-1) \theta}$ for all integers $a$ and $b, b \neq a+1$; hence knowing $k \theta$ for every nonzero $k$ suffices for complete knowledge of $(N,+)$. In order to assign, step by step, values to $c \theta$ for given integers $c$, we use a suitable function $\phi$ with $\phi^{n}=I$, where $n>1$ is an integer. An easy way of selecting such functions is developing them from functions of the form $r(x+q)^{-1}$ or $p x+r$ over some field, where $p=1$ or $-1, q$ and $r$ integers. Two examples will explain the method of construction.

3.1. The function $x \Phi=a-x$ over the real numbers, $a$ being an integral constant, satisfies $\Phi^{2 k}=I, k$ an arbitrary positive integer. The function can be "translated" into a function in $(N,+, \cdot)$ in various ways, for instance

$$
x f=a+x J,
$$

or if $x=J^{y}, a=J^{b}$, then $J^{y \phi}=J^{b}+J^{y+1}$ for $y \neq b$. Thus $J^{y \phi}=J^{b+(y-b) \theta}$ and $y \phi=b+(y-b) \theta$. We postulate now $\phi^{2 k}=I$ which cannot lead to a contradiction because then also $\Phi^{n}=I$, in the more special neofield of real numbers, would be contradictory. It can be easily verified that $y \phi^{2 k}=b+(y-b) \theta^{2 k}$, and if $\phi^{2 k}=I$, this becomes

$$
y-b=(y-b) \theta^{2 k}
$$


Now $\Phi^{2 k}=I$ is equivalent to $f^{2 k}=I$, and by developing this according to (26) we obtain

$$
x J^{2 k} L\left(a J^{2 k-1}\right) L\left(a J^{2 k-2}\right) \cdots L(a J) L(a)=x .
$$

If we construct a loop according to the rule (27) then the identity (28) will be valid in this loop. The actual construction will be described now for the case $b=3, k=2$. We assign the function values of $\theta$ stepwise in some specified order as, for instance, $0,1,-1,2,-2, \cdots$; but we omit values leading to coincidences and undefined values as $0 \theta, 0 \theta^{-1}, c \theta=c+1$. Also the period $2 k=4$ has to be observed. Proceeding in accordance with this rule we obtain $1 \theta=-1,1 \theta^{2}=(-1) \theta=2,1 \theta^{3}=2 \theta=-3,1 \theta^{4}=(-3) \theta=1,(-2) \theta=3,3 \theta=-4$, $(-4) \theta=4,4 \theta=-2$, etc.

This example yielded $(N,+)$ as an automorphic-inverse loop with the identity (28) which can be considered as a specialization of the left I.P. identity. If we had chosen some other order in assigning the values of $\theta$ we should have obtained another $(N,+)$ with the same properties, but in general these different $(N,+)$ 's are not isomorphic to each other.

3.2. As a second example we choose $x \Phi=(1-x)^{-1}$ over the real numbers $x \neq 1$. We have $\Phi^{3}=I$. A suitable "translation" of the function for $(N,+, \cdot)$ is

$$
x f=(1+x J)^{-1}
$$

or, with $x=J^{y}, J^{y \phi}=\left(1+J^{y+1}\right)^{-1}=J^{-y \theta}$,

$$
y \phi=-y \theta, \text { and } y \phi^{3}=y=(-(-y \theta) \theta) \theta .
$$

We postulate now $f^{3}=I$, according to $\Phi^{3}=I$. Thus we obtain

$$
(1+x J)+J=\left(\left(x+x^{2} J\right)+x J\right)+\left(x J+x^{2} J^{2}\right) .
$$

Our procedure would yield a loop $(N,+)$ with the identity $(31)$, but since the identity is rather cumbersome, we use an auxiliary condition. The function $\Phi$ satisfies evidently the relation $-x \cdot x \Phi \cdot x \Phi^{2}=1$, and we postulate as an analogous auxiliary condition

$$
x J \cdot x f \cdot x f^{2}=1
$$

which becomes, after substituting (29), $(1+x J)+J=x J$. This identity is equivalent to the C.I. property of the automorphic-inverse loop. The identity (31) follows from the C.I. property, thus it is consistent with it. From the identity (32) follows, with $x=J^{y}$,

$$
y+1-y \theta=(-y \theta) \theta
$$

as auxiliary condition which is consistent with (30). Now, with (30) and (33), the loop can be constructed along the lines indicated in 3.1. This construction of a C.I. loop is described in detail in [1]. 
3.3. Another example, not worked out here, is $x \Phi=x-1$ over a field of characteristic $k$. Obviously $\Phi^{k}=I$. The neofield "translation" that we choose is $x f=x+J$. This function yields an automorphic-inverse $(N,+)$ with the identity $x R(J)^{k}=x$.

All the neofields $(N,+, \cdot)$ thus constructed have the same properties that have been proved [6] for the case of 3.2: They are monogenic, their order is countable, their additive loops are simple; and by modifying the order of the steps by which the respective additive loop is constructed, uncountably many nonisomorphic loops $(N,+)$ can be produced [cf. 7].

\section{REFERENCES}

1. R. Artzy, On loops with a special property, Proc. Amer. Math. Soc. vol. 6 (1955) pp. $448-453$.

2. - A note on the automorphisms of special loops, Riveon Lematematika vol. 8 (1954) p. 81.

3. - Loops and generally situated 4-webs, Scientific Publications of Technion, Israel Institute of Technology vol. 6, 1954-1955, pp. 5-13.

4. R. H. Bruck, Contributions to the theory of loops, Trans. Amer. Math. Soc. vol. 60 (1946) pp. $245-354$.

5. - On a theorem of R. Moufang, Proc. Amer. Math. Soc. vol. 2 (1951) pp. 144-145.

6. - A Analogues of the ring of rational integers, Proc. Amer. Math. Soc. vol. 6 (1955) pp. $50-58$.

7. T. Evans, Some remarks on a paper by R. H. Bruck, Proc. Amer. Math. Soc. vol. 7 (1956) pp. 211-220.

ISRAEL INSTITUTE OF TECHNOLOGY, HAIFA, IsRaEL

UNIVERSITY OF WISCONSIN, Madison, Wis. 